\title{
Economic analysis of battery electric storage systems operating in electricity markets
}

\author{
F.-Javier Heredia \\ Group on Numerical Optimization \\ and Modeling (GNOM) \\ Universitat Politècnica de Catalunya - \\ BarcelonaTech \\ Jordi Girona 1-3, 08034 Barcelona. Spain \\ f.javier.heredia@upc.edu
}

\author{
Jordi Riera \\ Institut de Robòtica i Informàtica \\ Industrial (UPC/CSIC) \\ Llorens i Artigas 4, 08028 Barcelona, \\ riera@iri.upc.edu
}

\author{
Montserrat Mata, Joan Escuer and \\ Jordi Romeu \\ EMELCAT, S.L., Barcelona, Spain \\ direccio.tecnica@emelcat.cat
}

\begin{abstract}
Battery electric storage systems (BESS) in the range of 1-10 MWh is a key technology allowing a more efficient operation of small electricity market producer. The aim of this work is to assess the economic viability of Li-ion based BESS systems for small electricity producers. The results of the ex-post economic analysis performed with real data from the Iberian Electricity Market shows the economic viability of a Li-ion based BESS thanks to the optimal operation in day-ahead and ancillary electricity markets.
\end{abstract}

Index Terms-- battery energy storage systems, electricity markets, mathematical optimization, SAS/OR, wind power plants.

\section{INTRODUCTION}

The battery electricity storage systems (BESS) are mainly used as ancillary services or for supporting the large scale solar and wind integration in the existing power system, by providing grid stabilization, frequency regulation and wind and solar energy smoothing [1]. Medium size BESS in the range of 1-10 MWh is a key technology specially appropriate for small producers with non-dispatchable generator (wind power plants or PV) or almost non-dispatchable generation (co-generation). Lithium-ion (Li-ion) batteries provide high power and a large depth of discharge, fast charging and discharging capability and high round-trip efficiency [1] [2]. Despite the fact that the number of providers of ready to work Li-ion batteries has increased in the last years with prices that start to be competitive, some studies indicated that profits from energy arbitrage were insufficient to achieve capital cost recovery [3]. Nevertheless the same studies suggested the possibility for BESS to take advantage of other ancillary services to achieve economic viability. Following this idea a recent study [4] conclude that, under current UK market policy, it is still un-economical for a wind power plant (WPP) to use a Vanadium Redox Flow battery to provide the ancillary service of primary reserve. Indeed, BESS allow arbitrage in electricity markets as well as the participation in the ancillary services markets (as, for instance, reserve markets). We show in this work the economic viability of Li- ion based BESS through an ex-post analysis of the annual profit associated to the optimal operation of a BESS device in the day-ahed and secondary reserve markets of the Iberian Electricity Market (IEM). To this end we consider a Virtual Power Plant (VPP) comprising a generation unit, a BESS and some own consumption. A Mixed Integer Linear Programming problem (MILP) is proposed to find an approximation to the optimal operation of the VPP during a whole year. This MILP is used in the last part of this study to show the economic viability of a Li-ion based BESS associated to a WPP. Although the methodology presented in this study is applied to Li-ion batteries, it can be easily extrapolated to any other BESS technologies.

\section{MATHEMATICAL OPTIMIZATION MODEL}

\section{A. Acronyms}

AGC: automatic generation control.

BESS: battery electric storage system.

DAM: day-ahead market.

DOD: depth of discharge.

EM: electricity market.

EOL: end of life.

IEM: Iberian Electricity Market.

IPP: investment payback period.

ISO: independent system operator.

Li-ion: Lithium-ion

MILP: mixed integer linear programming.

PV: photovoltaic.

ROI: return of the investment.

SOC: state of charge.

SRB: secondary reserve band.

SRE: secondary reserve energy.

SRM: secondary reserve market.

SURE: secondary up reserve energy.

SDRE: secondary down reserve energy.

VPP: virtual power plant.

WPP: wind power plant.
The work of prof. F.-Javier Heredia was partially supported by grant MTM2013-48462-C2-1-R of the Ministry of Economy and Competitivity, Government of Spain. 


\section{B. Parameters}

$\mathcal{T}$ : $\quad$ set of hourly time periods of the year $\mathcal{T}=\{1,2, \ldots, 8760\}$.

$\mathcal{B}$ : set of identical batteries of the BESS.

cyc $^{\max }$ : battery's cycle life.

$C^{C A P}$ : capital cost of each battery [€].

$d^{\max }$ : battery's maximum charging/discharging rate $[k W]$.

$e^{\max }: \quad$ battery's capacity $[k W h]$.

$g_{t}^{\min }$ : minimum generation output $[k W h]$ at time period $t \in \mathcal{T}$.

$g_{t}^{\max }:$ maximum generation output at time period $t \in \mathcal{T}[k W h]$.

$L_{t}$ : own consumption of the VPP during period $t \in \mathcal{T}[k W h]$.

$\operatorname{soc}^{0}$ : SOC level at end-of-day.

soc $^{\text {min }}$ : minimum SOC.

soc ${ }^{\text {max }}$ : maximum SOC.

$\alpha^{S R}$ : ratio between the up/down band declared by the system operator.

$\beta_{t}^{U}, \beta_{t}^{D}: \quad$ fraction of the up/down reserve energy allocated to the VPP at time period $t \in \mathcal{T}$.

$\gamma^{R T E}: \quad$ round-trip efficiency.

$\delta^{R E P}:$ ratio of replacement cost to capital cost.

$\Delta t$ : length of the time periods [ $h]$ (1hour by default).

$\Delta t^{S R}$ : time response of the secondary reserve [hours] (15min by default).

$\lambda_{t}^{D A M}$ : $\quad$ DAM clearing price at time period $\in \mathcal{T}$ $[€ / k W h]$.

$\lambda_{t}^{S R B}: \quad$ SRB clearing price at time period $t \in \mathcal{T}$ $[€ / k W]$.

$\lambda_{t}^{S U R E}$ : SURE clearing price at time period $t \in$ $\mathcal{T}[€ / k W h]$.

$\lambda^{S D R E}: \quad$ SDRE clearing prices at time period $\in \mathcal{T}$ $[€ / k W h]$.

\section{Decision variables}

$m_{t}$ : VPP's price accepting bid to the DAM at time period $t \in \mathcal{T}[k W h]$.

$g_{t}$ : VPP's generation at time period $\in \mathcal{T}[k W]$.

$c_{b t}$ : charging rate of the battery $b \in \mathcal{B}$ at time period $t \in \mathcal{T}[\mathrm{kW}]$.

$d_{b t}$ : discharging rate of the battery $b \in \mathcal{B}$ at time period $t \in \mathcal{T} \quad[k W]$.

$i d_{t}$ : binary variable establishing if the BESS is discharging $\left(i d_{t}=1\right)$ or charging $\left(i d_{t}=0\right)$ at time period $t \in \mathcal{T}$.

$\operatorname{soc}_{b t}$ : SOC of battery $b \in \mathcal{B}$ at the end of time period $t \in \mathcal{T} \cup\{0\}$.

$r_{b t}^{U}, r_{b t}^{D}$ : up/down secondary reserve of battery $b \in \mathcal{B}$ at time period $t \in \mathcal{T}[\mathrm{kW}]$.

$e_{b t}^{U}, e_{b t}^{D}: \quad$ up/down secondary reserve energy allocated to the battery $b \in \mathcal{B}$ at time period $t \in \mathcal{T}[k W h]$.

\section{Functions:}

$e_{b t}(\cdot)$ : stored energy of battery $b \in \mathcal{B}$ at the end of period $t \in \mathcal{T}[\mathrm{kWh}]$.

$\operatorname{cyc}(\cdot):$ number of charge/discharge cycles of a given BESS operation policy.
$R^{E M}(\cdot)$ : electricity markets revenues $[€]$.

$C^{G}(\cdot)$ : generation costs of the VPP [€].

$C^{L C C}(\cdot)$ : life cycle costs of the BESS [€].

$E P^{V P P}(\cdot):$ estimated profit of the VPP [€].

$E R^{D A M}(\cdot)$ : estimated revenues from the DAM.

$E R^{S R B}(\cdot)$ : estimated revenues from the SRB market.

$A P^{V P P}(\cdot)$ : annual profit of the VPP [€].

$A R^{D A M}(\cdot)$ : DAM's annual revenues [ $\left.€\right]$.

$A R^{S R B}(\cdot)$ : SRB's annual revenues [€].

$A R^{S R E}(\cdot)$ : SRE's annual revenues/payments [€].

$\triangle P^{B E S S}(\cdot):$ increase of profit due to BESS [€].

$I P P(\cdot)$ : investment payback period [€].

$F E O L(\cdot)$ : EOL of the first exhausted battery.

$R O I(\cdot)$ : return of the investment $[€]$.

\section{E. Day-ahead market.}

We are assuming that the VPP submits a price accepting bid of $m_{t} k W h$ to the DAM. Consequently, all the energy $m_{t}$ will be matched and remunerated at the DAM's clearing price $\lambda_{t}^{D A M}$. The expression of the amount of energy auctioned to the DAM at time period $t$ is

$$
m_{t}=g_{t}+\Delta t \cdot \sum_{b \in \mathcal{B}}\left(d_{b t}-c_{b t}\right)-L_{t} \quad t \in \mathcal{T}
$$

where variable $m_{t}$ represents the amount of energy sold ( $>0$ ) or purchased $(<0)$ to the day-ahead market. $g_{t}$ is the joint production of the VPP's generation units. $\Delta t \cdot d_{b t}$ and $\Delta t \cdot c_{b t}$ are, respectively, the discharged/charged energy of each individual battery of the BESS, and $L_{t}$ the own consumption of the VPP, if any. The VPP's generation is restricted to be within some operational limits:

$$
g_{t}^{\min } \leq g_{t} \leq g_{t}^{\max } \quad t \in \mathcal{T}
$$

with, possibly, $g_{t}^{\min }=g_{t}^{\max }$ at some (or all) time periods if the generation unit of the VPP is not fully dispatchable. The bounds on variables $c_{t}$ and $d_{t}$ will be discussed later on. The sign of variable $m_{t}$ depends on whether the VPP is limited to act in the EM as a producer, as a consumer or can assume both roles $^{1}$

$$
\left\{\begin{array}{lc}
m_{t} \geq 0 & \text { if VPP is a producer } \\
m_{t} \text { free } & \text { if VPP is a producer/consumer } \\
m_{t} \leq 0 & \text { if VPP is a consumer }
\end{array} \quad t \in \mathcal{T}\right.
$$

\section{F. Battery electric storage system.}

Constraints (4)-(6) define the bounds and coupling of variables $c_{b t}$ and $d_{b t}$ with the help of the binary variable $i d_{t}$

$$
\begin{array}{cc}
0 \leq d_{b t} \leq d^{\text {max }} \cdot i d_{t} & b \in \mathcal{B}, t \in \mathcal{T} \\
0 \leq c_{b t} \leq d^{\text {max }} \cdot\left(1-i d_{t}\right) & b \in \mathcal{B}, t \in \mathcal{T} \\
i d_{t} \in\{0,1\} & t \in \mathcal{T}
\end{array}
$$

These constraints prevent the BESS to charge and discharge simultaneously at the same time period $t$. Moreover our model

${ }^{1}$ For instance, through the association of the VPP with two different generic units. 
assumes that all the batteries of the BESS has the same charge/discharge state at every time period $t$.

The state of charge (SOC) of each battery along all the time periods can be traced through the next two set of constraints

$$
\begin{array}{rlrl}
\operatorname{soc}_{b t} & =\operatorname{soc}_{b, t-1} & \\
& +\Delta t \cdot\left(c_{b t}-d_{b t} / \gamma^{R T E}\right) / e^{\max } & b \in \mathcal{B}, t \in \mathcal{T} \\
& +\left(e_{b t}^{D}-e_{b t}^{U} / \gamma^{R T E}\right) / e^{\max } & & \\
\operatorname{soc}^{\min } \leq \operatorname{soc}_{b t} \leq \operatorname{soc}^{\max } & b \in \mathcal{B}, t \in \mathcal{T}
\end{array}
$$

where $e_{b t}^{D}$ and $e_{b t}^{U}$ corresponds to extra charge/discharge (resp.) of the secondary reserve energy allocated by the ISO (see section $\mathrm{G}$ ). Coefficient $\gamma^{R T E}$ is the round-trip efficiency of the battery ${ }^{2}$. Finally, to obtain a solution representing a VPP operation that could actually be implemented we force the SOC at the end of every day to match a fixed prestablished level $\operatorname{soc}^{0}$

$$
\operatorname{soc}_{b t}=\operatorname{soc}^{0} \quad b \in \mathcal{B}, t=k \cdot 24, k \in \mathbb{N}^{0} .
$$

This is equivalent to consider a day-ahead optimization of the VPP where at day D-1 the optimal operation during day D is decided. Should (9) be disregarded the model will be allowed to perform a "more than one day-ahead" optimization, anticipating unknown information at day D-1, with the effect of an overestimation of the profits. Conversely, to include (9) means to probably underestimate the potential benefits of the BESS, but we prefer this last approach to avoid being too much optimistic about the advantages of the BESS.

\section{G. Secondary reserve market.}

Our model assumes that the VPP submits a price accepting bid to the SRM equivalent to the total available reserve up and reserve down of all the batteries in the BESS. Let $r_{b t}^{U}$ and $r_{b t}^{D}$ be respectively the contribution of battery $b \in \mathcal{B}$ to the VPP's up and down reserve. At each time period $t$ the available amount of up and down reserve depends on the state of the battery. First, the battery's reserve is limited by the gap between the maximum and the current charging/discharging rate:

$$
\begin{array}{ll}
0 \leq r_{b t}^{U} \leq d^{\text {max }}-d_{b t}+c_{b t} & b \in \mathcal{B}, t \in \mathcal{T} \\
0 \leq r_{b t}^{D} \leq d^{\text {max }}+d_{b t}-c_{b t} & b \in \mathcal{B}, t \in \mathcal{T}
\end{array}
$$

Second, the battery's capacity $e^{\max }$ imposes additional limits to the reserve. The total stored energy at the beginning of time period $t$ is equal to $\operatorname{soc}_{b, t-1} \cdot e^{\max }$. The remaining energy at the end of this same period after a charge/discharge of $c_{b t} / d_{b t}$ will be

$$
\begin{aligned}
e_{b t}\left(\operatorname{soc}_{b, t-1}, c_{b t}, d_{b t}\right)= & \operatorname{soc}_{b, t-1} \cdot e^{\max } \\
& +\Delta t \cdot\left(c_{b t}-d_{b t} / \gamma^{R T E}\right) .
\end{aligned}
$$

2 Ratio of the total energy storage system output (discharge) divided by total energy input (charge).
To allocate a given amount $r_{b t}^{U} k W$ of up reserve would mean to drain the battery by $\Delta t^{S R} \cdot r_{b t}^{U} / \gamma^{R T E}$ additional $k W h$, and the actual stored energy at the end of period $t$ will be

$$
\begin{aligned}
& e_{b t}\left(\operatorname{soc}_{b, t-1}, c_{b t}, d_{b t}, r_{b t}^{U}\right)=\operatorname{soc}_{b, t-1} \cdot e^{\max } \\
& +\Delta t \cdot\left(c_{b t}-d_{b t} / \gamma^{R T E}\right) \\
& -\Delta t^{S R} \cdot r_{b t}^{U} / \gamma^{R T E}
\end{aligned}
$$

The reduction in the total stored energy (13) is bounded below by the minimum SOC

$$
\begin{aligned}
& \operatorname{soc}_{b, t-1} \\
& +\Delta t \cdot\left(c_{b t}-d_{b t} / \gamma^{R T E}\right) / e^{\max } \quad b \in \mathcal{B}, t \in \mathcal{T} \\
& -\Delta t^{S R} \cdot r_{b t}^{U} /\left(\gamma^{R T E} \cdot e^{\max }\right) \geq \operatorname{soc}^{\min }
\end{aligned}
$$

Analogously, any allocation of down reserve to the VPP implies some extra charging of the batteries that increases the total stored energy by $\Delta t^{S R} \cdot r_{b t}^{D} k W h$. The total stored energy is then bounded above by the maximum SOC:

$$
\begin{aligned}
& \operatorname{SOc}_{b, t-1} \\
& +\Delta t \cdot\left(c_{b t}-d_{b t} / \gamma^{R T E}\right) / e^{\max } \quad b \in \mathcal{B}, t \in \mathcal{T} \\
& +\Delta t^{S R} \cdot r_{b t}^{D} / e^{\max } \leq \operatorname{soc}^{\max }
\end{aligned}
$$

The up and down secondary reserve band bids must satisfy the relation $\alpha^{S R}$ declared by the ISO:

$$
\sum_{b \in \mathcal{B}} r_{t}^{U}=\alpha^{S R} \cdot \sum_{b \in \mathcal{B}} r_{t}^{D} \quad t \in \mathcal{T}
$$

Equations (10)- (11) and (14)-(16) defines the total amount of the available up/down secondary reserve band of the VPP at time period $t$. As for the case of the day-ahead markets, we assume that the VPP submits all its available reserve as an accepting bid to the SRM. Let's assume that only a given fraction $\beta_{t}^{U}$ and $\beta_{t}^{D}$, of the total reserve band bid will be eventually allocated during the AGC to the VPP (see section III for further details on this coefficients). The down reserve energy, $e_{b t}^{D}$, represents a decrease of the VPP's total net production attained through an extra charge of the battery:

$$
e_{b t}^{D}=\Delta t^{S R} \cdot \beta_{t}^{D} \cdot r_{t}^{D} \quad b \in \mathcal{B}, t \in \mathcal{T}
$$

Conversely, the up reserve energy, $e_{t}^{U}$, corresponds to an increase of the VPP's production that forces an extra drainage of the BESS:

$$
e_{b t}^{U}=\Delta t^{S R} \cdot \beta_{t}^{U} \cdot r_{b t}^{U} \quad b \in \mathcal{B}, t \in \mathcal{T}
$$

\section{H. VPP's annual profit estimation}

The objective function represents the total profit of the VPP as the difference between incomes and expenditures during the whole optimization horizon (typically one year). Incomes are the revenues from the day-ahead and secondary reserve market while expenses come from the generation costs (if any) and the BESS's wear costs. Our ex-post analysis aims at approximate as fair as possible the situation of a VPP manager that has to decide on day $D-1$ the optimal bid of the VPP to the day-ahead and reserve markets for the next day $D$ (comprising periods $t^{\prime}, t^{\prime}+1, \ldots, t^{\prime}+24$ ) without knowing 
the true value of the day-ahead and reserve markets clearing prices $\left\{\lambda_{t}^{D A M}, \lambda_{t}^{S R B}\right\}, t=t^{\prime}, t^{\prime}+1, \ldots, t^{\prime}+24$. Then, instead of computing the market revenues with the true value of the market clearing prices at day $\mathrm{D}$, that would be equivalent to assume that the VPP manager has a priori knowledge of the clearing prices, we use the prices of the same weekday of the previous week, that is $\left\{\lambda_{t-168}^{D A M}, \lambda_{t-168}^{S R B}\right\}, t=t^{\prime}, t^{\prime}+1, \ldots, t^{\prime}+$ 24 , which is an information available at day $D-1$. This is equivalent to assume that at day $D-1$ the VPP manager finds the optimal bid with respect to the one-week-ago clearing prices. This assumption results in the following estimated market revenue functions

$$
\begin{aligned}
E R^{D A M}(m) & =\sum_{t \in \mathcal{T}} \lambda_{t-168}^{D A M} \cdot m_{t} \\
E R^{S R B}\left(r^{U}, r^{D}\right) & =\sum_{b \in \mathcal{B}, t \in \mathcal{T}} \lambda_{t-168}^{S R B} \cdot\left(r_{b t}^{U}+r_{b t}^{D}\right)
\end{aligned}
$$

This one-week-ago substitution in the objective function of the clearing market prices allows to obtain the optimal VPP operation without any priori knowledge of the market clearing prices. The actual revenues of the VPP are $\sum_{t \in \mathcal{T}} \lambda_{t}^{D A M} \cdot m_{t}+$ $\sum_{t \in \mathcal{T}} \lambda_{t}^{S R B} \cdot\left(r_{t}^{U}+r_{t}^{D}\right)$, and this will be the expression used to analyse the economic benefits of the BESS in section III. The incomes/payments associated to the secondary reserve energy are not included in the objective functions because they depend on the AGC of the ISO and consequently they use to be disregarded in the optimization. Nevertheless the extra incomes $\sum_{b \in \mathcal{B}, t \in \mathcal{T}} \lambda_{t}^{S U R E} \cdot e_{b t}^{U}$ and payments $\sum_{b \in \mathcal{B}, t \in \mathcal{T}} \lambda_{t}^{S D R E}$. $e_{b t}^{D}$ will be taken into account when calculating the actual value of the total annual profits of the VPP.

Let $C^{G}(g)$ represent the operation costs of the generation unit of the VPP. Depending on the characteristics of the generation unit $C^{G}(g)$ uses to be considered linear, piece-wise linear or quadratic. Besides the generation costs, in order to do an accurate study of the economic viability of the BESS the operation cost associated to the charge/discharge cycles of the Li-ion battery must be taken into account. End of life (EOL) of a Li-ion battery is usually considered to happen when the capacity loss is between $20 \%-30 \%$ [4]. The cycle life cyc max $^{\text {max }}$ of a battery is the estimated maximum number of complete charge/discharge processes (cycles) before reaching EOL. The cycle life depends on several factors (temperature, charging rate,...) but above all, the maximum and minimum SOC (expressed usually in terms of the depth of charge, DOD $=1$ SOC). Let $c y c^{\max }$ be the cycle life associated to some pair $\left(\operatorname{soc}^{\min }\right.$, soc $\left.^{\max }\right)$. The total number of cycles performed during period $\mathcal{T}$ by the battery $b$ is

$$
\operatorname{cyc}\left(c_{b}, e_{b}^{D}\right)=\frac{\Delta t \cdot \sum_{b \in \mathcal{B}, t \in \mathcal{T}} c_{b t}+\sum_{b \in \mathcal{B}, \in \mathcal{T}} e_{b t}^{D}}{e^{\max }}
$$

where the numerator is the total energy charged along all the time horizon $\mathcal{T}$. Then, if the battery has been operated satisfying $\operatorname{soc}^{\min } \leq \operatorname{soc}_{t} \leq \operatorname{soc}^{\max }$ (guaranteed by constraints (8)), the life cycle cost during $\mathcal{T}$ of the BESS is

$$
C^{L C C}\left(c, e^{D}\right)=\delta^{R E P} \cdot C^{C A P} \cdot \sum_{b \in \mathcal{B}} c y c\left(c_{b}, e_{b}^{D}\right) / c y c^{\max }
$$

where $C^{C A P}$ stands for the capital $\operatorname{cost}^{3}$ of each individual battery and $\delta^{R E P}$ the fraction of the capital cost that corresponds to the replacement of the exhausted battery by a new one at its EOL after reaching $c y c^{\max }$ cycles. Finally, the estimated profit of the VPP is the difference between total estimated revenues and total costs

$$
\begin{aligned}
& E P^{V P P}\left(m, r^{U}, r^{D}, g, c, e^{D}\right)= \\
& E R^{D A M}(m)+E R^{S R B}\left(r^{U}, r^{D}\right)-C^{G}(g)-C^{L C C}\left(c, e^{D}\right) .
\end{aligned}
$$

The role of the life cycle cost term $C^{L C C}\left(c, e^{D}\right)$ is to penalise the excess of cycles, but this term must be disregarded in the calculation of the total annual profits due to the BESS.

\section{The (BESSEM) optimization model.}

The constraints and objective function developed in the precedent sections defines the so called Battery Electricity Storage System in Electricity Markets model (BESSEM):

$$
(B E S S E M)\left\{\begin{array}{cc}
\max & E P^{V P P}\left(m, r^{U}, r^{D}, g, c, e^{D}\right) \\
\text { s.t.: } & (1)-(3) \\
\text { DAM : } & (4)-(9) \\
\text { BESS : } & (10),(11),(14)-(18)
\end{array}\right.
$$

(BESSEM) model is a large scale mixed integer linear or quadratic (depending on $C^{G}(g)$ ) optimization problem, with $2 \cdot|\mathcal{T}|+7 \cdot|\mathcal{T}| \cdot|\mathcal{B}|=17520+61320 \cdot|\mathcal{B}| \quad$ continuous variables, $|\mathcal{T}|=8760$ binary variables and $2 \cdot|\mathcal{T}|+10 \cdot$ $|\mathcal{T}| \cdot|\mathcal{B}|=17520+87600 \cdot|\mathcal{B}|$ linear constraints. It must be noticed that actually problem (BESSEM) is decomposable in 365 daily independent subproblems due to constraints (9).

Let $x \in \mathbb{R}^{2 \cdot|\mathcal{T}|+7 \cdot|\mathcal{T}| \cdot|\mathcal{B}|} \times\{0,1\}^{|\mathcal{T}|}$ represent the vector of decision variables of model (BESSEM). Actually, the optimal solution $\quad x^{*} \stackrel{\text { def }}{=} \operatorname{argmax}\{(B E S S E M)\}$ is a suboptimal approximation of the optimal operation of the VPP because it could be easily improved in the real-time management ${ }^{4}$. The actual annual profit earned by the VPP is

$$
\begin{gathered}
A P^{V P P}\left(x^{*}\right)= \\
\underbrace{\sum_{t \in \mathcal{T}} \lambda_{t}^{D A M} \cdot m_{t}^{*}}_{A R^{D A M}\left(m^{*}\right)}+\underbrace{\sum_{b \in \mathcal{B}, t \in \mathcal{T}} \lambda_{t}^{S R B} \cdot\left(r_{b t}^{U^{*}}+r_{b t}^{D^{*}}\right)}_{A R^{S R B}\left(r^{U^{*}}, r^{D^{*}}\right)} \\
+\underbrace{\sum_{-C^{G}\left(g^{*}\right)}\left(\lambda_{t}^{S U R E} \cdot e_{b t}^{U^{*}}-\lambda_{t}^{S D R E} \cdot e_{b t}^{D}\right)}_{A R^{S R E}\left(e^{U^{*}}, e^{D^{*}}\right)}
\end{gathered}
$$

where (24) are the actual incomes from the DAM and SRB market, the terms in (25) are the incomes/payments of the secondary up and down reserve energy and (26) is the generation cost.

\footnotetext{
${ }^{3}$ Equipment capital and first installation

${ }^{4}$ For instance, with a better forecasting of $\lambda^{D A M}$ and $\lambda^{D R M}$ or relaxing constraints (9).
} 


\section{CASE STUDY: ECONOMIC VIABILITY OF A BESS ATTACHED TO A WPP}

The aim of this last part of the study is to show the potential of model $(B E S S E M)$ as a tool to analyse the economic viability of the BESS. Let's consider a VPP comprising a WPP (non-dispatchable production $g_{t}=g_{t}^{\text {min }}=$ $g_{t}^{\max }, m_{t} \geq 0 \mathrm{kWh}, C^{G}(g)=0, L_{t}=0 \mathrm{kWh}, t \in \mathcal{T}$, see TABLE I) and a BESS including six identical Li-ion batteries $(|\mathcal{B}|=6)$ with a total capacity of $6 M W h$ and $3 M W$ of discharge power (see TABLE II).

TABLE I : WPP GENERATION (REAL DATA FOR 2013).

\begin{aligned} & $\max \left\{g_{t}\right\}= 5464 \mathrm{kWh} \\ &$\hline$E\left[g_{t}\right]=1541 \mathrm{kWh} \\ &$\hline$\sigma=1978 \mathrm{kWh} \\ &$\hline\end{aligned}

TABLE II : PARAMETERS OF THE LI-ION BATTERIES (BASED ON [2] AND [5]).

\begin{aligned} & \hline $\mathcal{B}=\{1,2, \ldots, 6\} \\ &$\hline$d^{\max }=500 \mathrm{~kW} \\ &$\hline$e^{\max }=1000 \mathrm{kWh} \\ &$\hline$C^{C A P}=1250 \frac{\mathrm{kWh}}{\mathrm{kWh}} \cdot \mathrm{e}^{\max } \\ &$\hline$c y c^{\max }=6000 \\ &$\hline\end{aligned}

\begin{tabular}{|c|c|}
\hline$\delta^{R E P}=$ & 0.6 \\
\hline $\operatorname{soc}^{0}=$ & 0.5 \\
\hline $\operatorname{soc}^{\max }=$ & 0.9 \\
\hline $\operatorname{soc}^{\min }=$ & 0.3 \\
\hline$\gamma^{R T E}=$ & 0.8 \\
\hline
\end{tabular}

The VPP participates in the DAM and SRM of the Iberian Electricity Market. The electricity market prices considered in this study $\left(\lambda^{D A M}, \lambda^{S R B}, \lambda^{S U R E}, \lambda^{S D R E}\right)$ are the actual prices during year 2013 of the IEM, available at the websites of the IMO and ISO ( [6] and [7] resp.). The SRM time response is $15 \min \left(\Delta \mathrm{t}^{S R}=0.25 h\right)$ and the up/down reserve ratio $\alpha^{S R}=1.5$. Coefficients $\beta_{t}^{U}$ and $\beta_{t}^{D}$ was randomly computed before the optimization in a way that i) at most only one of them are nonzero for a given $t$, ii) there is some reserve energy requirement in the $70 \%$ of the time periods (approx.) and iii) this requirement is always greater than the $70 \%$ of the submitted up and down reserve. The associated (BESSEM) model has 385440 continuous variables, 8760 binary variables and 573120 linear constraints. The SAS/OR 9.3 ${ }^{\circledR}$ software [8] was used to implement this MILP problem in the algebraic modelling language of the OPTMODEL procedure and solved in 4 minutes with the built-in branch-and-cut optimizer MILP on a desktop PC (i7@2.93GHz, 8GB RAM) under Windows 7 Professional. TABLE III shows the main results obtained with the $(B E S S E M)$ model.

\section{CONCLUSIONS}

A MILP optimization model, (BESSEM) has been formulated to assess the economic viability of BESS embedded in a VPP that bids to both the DAM and the SRM. This model has been used to analyse a $6 M W h / 3 M W \mathrm{Li}$-ion BESS attached to a real WPP that operates in the IEM with real data of year 2013. The numerical results shows the profitability of the BESS, with an investment payback period of 10 years, a 20 years EOL and a return of the investment (ROI) of $100 \%$. It is to mention that this high ROI is a consequence of the high annual revenues of the BESS's reserve capacity in the secondary reserve market. The ex-post analysis of the $(B E S S E M)$ model gives a general methodology that can be easily applied to any BESS technology and extended to incorporate other electricity markets as, for instance, intra-day markets.
TABLE III : ECONOMIC ANALYSIS.

\begin{tabular}{|c|c|c|}
\hline DAM's annual revenue : & $A R^{D A M}\left(m^{*}\right)=$ & $529,430 €$ \\
\hline SRM's annual revenue : & $A R^{S R B}\left(r^{U^{*}}, r^{U^{*}}\right)=$ & $736,258 €$ \\
\hline SRE's annual revenue : & $A R^{S R E}\left(e^{U^{*}}, e^{U^{*}}\right)=$ & $41,896 €$ \\
\hline Annual profit of the VPP : & $A P^{V P P}\left(x^{*}\right)=$ & $1,307,584 €$ \\
\hline Annual profit of the WPP ${ }^{(a)}$ : & $A P^{W P P}=$ & $569,798 €$ \\
\hline $\begin{array}{l}\text { Increase of profit due to the } \\
\qquad \text { BESS }^{(\mathrm{b})} \text { : }\end{array}$ & $\Delta P^{B E S S}\left(x^{*}\right)=$ & $737,786 €$ \\
\hline Capital cost of the BESS $^{(\mathrm{c})}$ : & $C^{B E S S}=$ & $7,500,000 €$ \\
\hline Investment payback period $^{(\mathrm{d})}$ & *) $=$ & 10.17 years \\
\hline First end of life $^{(\mathrm{e})}$ : & $F E O L\left(x^{*}\right)=$ & 20.35 years \\
\hline Return of the investment ${ }^{(\mathrm{t})}$ : & $R O I\left(x^{*}\right)=$ & $100 \%$ \\
\hline
\end{tabular}

(a) : $\quad A P^{W P P}=\sum_{t \in \mathcal{T}} \lambda_{t}^{D A M} \cdot g_{t}$

(b) : $\quad \triangle P^{B E S S}\left(x^{*}\right)=A P^{V P P}\left(x^{*}\right)-A P^{W P P}$

(c) : $\quad C^{B E S S}=|\mathcal{B}| \cdot C^{C A P}$

(d) : $\operatorname{IPP}\left(x^{*}\right)=C^{B E S S} / \triangle P^{B E S S}\left(x^{*}\right)$

(e) : EOL of the first exhausted battery: FEOL $\left(x^{*}\right)=c y c^{\max } / \max _{b \in \mathcal{B}}\left\{c y c\left(c_{b}^{*}, e_{b}^{D^{*}}\right)\right\}$.

(f) : $\quad R O I\left(x^{*}\right)=\left(\operatorname{EOL}\left(c^{*}, e^{D^{*}}\right) \cdot \Delta P^{B E S S}\left(x^{*}\right)-C^{B E S S}\right) / C^{B E S S}$

\section{REFERENCES}

[1] A. Poullikkas, "A comparative overview of large-scale battery systems," Renewable and Sustainable Energy Reviews, vol. 27, pp. 778-788, 2013.

[2] F. Díaz-González, A. Sumper, O. Gomis-Bellmunt and R. Villafáfila-Robles, "A review of energy storage technologies for wind power applications,” Renewable and Sustainable Energy Reviews, vol. 16, pp. 2154-2171, 2012.

[3] M. Kintner-Meyer, P. Balducci, W. Colella, C. Jin, T. Nguyen, V. Viswanathan y Y. Zhang, «National Assessment of Energy Storage for Grid Balancing and Arbitrage: Phase 1, WECC,» Richland, 2012.

[4] L. Johnston, F. Díaz-González, O. Gomis-Bellmunt, C. Corchero-García y M. Cruz-Zambrano, «Methodology for the economic optimisation of energy storage systems for frequency support in wind power plants,» Applied Energy, $n^{\circ}$ 137, pp. 660-669, 2015.

[5] Saft Batteries, “Lithium-ion battery life - Saft," [Online]. Available:

http://www.saftbatteries.com/force_download/li_ion_batter y_life_TechnicalSheet_en_0514_Protected.pdf. [Accessed 31 January 2015].

[6] “omie,” Operador del Mercado Ibérico de Electricidad, [Online]. Available: http://www.omie.es. [Accessed 62 2015].

[7] “e·sios," Red Eléctrica de España, [Online]. Available: http://www.esios.ree.es/. [Accessed 0602 2015].

[8] "SAS/OR," SAS Institute Inc., [Online]. Available: http://www.sas.com/en_us/software/analytics/sas-or.html. [Accessed 0602 2015]. 
